\title{
Efficacy of Altered Two-Point Fixation in Zygomaticomaxillary Complex Fracture
}

\author{
Jun Hyeok Kim (iD, Ye Sol Kim, Deuk Young Oh, Young Joon Jun, Jong Won Rhie, \\ and Suk-Ho Moon \\ Department of Plastic \& Reconstructive Surgery, College of Medicine, The Catholic University of Korea, Seoul, Republic of Korea \\ Correspondence should be addressed to Suk-Ho Moon; nasuko@catholic.ac.kr
}

Received 18 November 2019; Revised 10 January 2020; Accepted 27 January 2020; Published 18 March 2020

Academic Editor: Jiang Du

Copyright (c) 2020 Jun Hyeok Kim et al. This is an open access article distributed under the Creative Commons Attribution License, which permits unrestricted use, distribution, and reproduction in any medium, provided the original work is properly cited.

\begin{abstract}
Purpose. To reconstruct a zygomaticomaxillary complex (ZMC) fracture, zygomaticofrontal (ZF) suture is the most reliable site to assess anatomical alignment and to secure rigidity. It has been chosen primary site to be fixed, but approach through the lateral eyebrow incision may leave a visible scar. This study suggests altered two-point fixation of ZMC fracture without accessing the ZF suture. Methods. In the retrospective study, a total of 40 patients with ZMC fracture were divided into two groups (group 1, twopoint fixation and group 2, three-point fixation). Patient demographics and follow-up were evaluated, and degree of reduction including cortical gaps of ZF and inferior orbital (IO) area, protruding difference of zygoma, and malar difference using asymmetry index were measured through preoperative and postoperative CT. Results. Preoperatively, the means of ZF displacement, IO displacement, protruding difference of zygoma, and facial asymmetry index between the groups were not statistically different. The result was the same after the operation. However, all variables were significantly different before and after surgery within each group. Moreover, mean operation time was significantly different between groups $(P$ value $=0.026)$. Conclusion. Altered two-point fixation in ZMC fracture excluding incision approaching the ZF provides surgical efficacy and similar surgical outcomes to three-point fixation but offers reduced operation time and fewer complications.
\end{abstract}

\section{Introduction}

Facial appearance affects the foundation of an individual's personality, and facial change due to injury can cause harmful alteration in one's sense of self and how one interacts and expresses oneself in society [1]. The zygomatic bone is the most prominent and characteristic in the midface [2], and its traumatic fracture may lead to crucial deformity of the face $[3,4]$. It is essential to restore the bony structure of the zygoma to its original shape.

The standard treatment for zygomaticomaxillary complex (ZMC) fracture has been open reduction and internal fixation (ORIF) [5], and sites of one-, two-, or three-point fixation are selected based on stability of the fractured zygoma $[6,7]$. Among fixation sites, the zygomaticofrontal (ZF) suture followed by zygomaticosphenoidal (ZS) suture has been the single most reliable site for anatomical alignment and secure fixation $[8,9]$. Thus, this site has been the primary location of fixation $[2,5,6,10-13]$ even in 1point fixation $[14,15]$. However, the ORIF approach of ZF suture through a lateral eyebrow incision may leave visible scars, uncomfortable palpability of plates on thin skin, and risk of drill penetration into the anterior cranial fossa $[8,12,16]$.

Thus, this study compares the results of altered twopoint fixation at the zygomaticomaxillary (ZM) buttress and infraorbital rim (IO) with three-point fixation with a ZF suture in ZMC fracture.

\section{Patients and Methods}

In this single-center, retrospective study, we evaluated the medical records and three-dimensional (3D) computed 
tomography (CT) scans of 117 patients with type B of ZMC fracture [17] between December 2015 and April 2019.

The inclusion criteria were as follows:

(1) Diagnosis of unilateral ZMC tetrapod fracture with preoperative radiological evaluation including $3 \mathrm{D}$ CT

(2) ORIF within two weeks after injury

(3) Postoperative evaluation including clinical outcomes and radiological examination including $3 \mathrm{D} C \mathrm{CT}$ within three months postoperative.

The exclusion criteria were as follows:

(1) Only fracture of the zygomatic arch (case of closed reduction, type A injuries) [17]

(2) Complex or combined fracture needing ORIF such as fracture of the mandible or frontal bone as well as type $\mathrm{C}$ injuries [17]

(3) No preoperative or postoperative 3D CT scans

(4) No postoperative evaluation (because the patient did not visit the outpatient clinic of the Department of Plastic and Reconstructive Surgery of our medical institution around three months postoperatively).

The present study was approved by the institutional review board (IRB) of our medical institution (IRB approval number: KC19RESI0427). The requirement for informed consent was waived due to the retrospective nature of the study.

A total of 40 patients was included in this study and divided into two groups. Group 1 was composed of 20 patients who underwent two-point (ZM buttress and IO area) ORIF through buccogingival and subciliary incisions, and group 2 comprised 20 patients who underwent three-point (ZM buttress, IO, and ZF areas) ORIF through buccogingival, subciliary, and lateral eyebrow incisions.

2.1. Operative Technique. The fracture sites were exposed including the fracture line so that plates could be applied under general anesthesia. The ZM area was exposed approximately $1.0 \mathrm{~cm}$ from the infraorbital rim for insertion of $6 \mathrm{~mm}$ dual-top screws (Jeil Medical Corporation, Seoul, Republic of Korea) perpendicular to the direction of reduction. The screw was inserted, and a 26-gauge wire was passed through a hole in the screw head. Displaced fragments were reduced anatomically by retracting the wire. If a bone fragment including the zygomatic arch was insufficiently reduced, another screw was inserted on the ZM buttress, and the two screws were retracted for anatomical reduction. In this process, the indicators of accurate reduction were concave alignment of the orbital floor, straightened continuity of the infraorbital rim, and palpation of the lateral rim for group 1 and alignment of the greater wing of the ZS for group 2. After confirming anatomical reduction, each fracture site was fixed with absorbable plates and screws (Inion CPS, Tampere, Finland). The wound was thoroughly irrigated, and hemostasis was confirmed. The periosteum, skin, and mucosa were closed in a layer-by-layer manner.

2.2. Management. Patients consumed a liquid diet for three days after surgery. They could then eat a general diet composed of soft food for six months. After two months, 3D CT scan was performed to confirm correct alignment and maintenance of bone fragments.

2.3. Measurement. Patient demographics and follow-up data were evaluated, and the degree of reduction including cortical gaps of ZF and IO area (Figure 1), protruding difference of zygoma, and malar difference using asymmetry index were measured via preoperative and postoperative 3D CT. The protruding difference of zygoma was compared by measuring the distance from the most prominent point of each zygomatic arch to A line. (A line: a virtual line from the pyriform aperture to the condyle of the mandible) (Figure 2). The asymmetry index was calculated using the following formula to compare the difference in prominence of the zygomatic arches $[18,19]$ (Figure 3):

Asymmetry index $=\sqrt{(\mathrm{Hr}-\mathrm{Hl})^{2}+(\mathrm{Vr}-\mathrm{Vl})^{2}+(\mathrm{Dr}-\mathrm{Dl})^{2}}$,

where $\mathrm{Hr}$ is right horizontal length, $\mathrm{Hl}$ is left horizontal length, Vr is right vertical length, Vl is left vertical length, Dr is right distance from midpoint, and $\mathrm{Dl}$ is left distance from midpoint.

2.4. Statistical Analysis. For nominal variables, fractions in percentages were calculated, and Fisher's exact test was used for comparison. For continuous variables, the mean and SD were used for description, and the difference between groups was compared using Mann-Whitney test or paired $T$ test. $P$ value less than 0.05 indicated a statistically significant difference.

\section{Results}

The baseline characteristics and demographic data of the patients are summarized in Table 1. The groups had no differences in age, sex, lesion side, causes of trauma, concomitant injuries, and operation delay. Preoperatively, the means of ZF displacement, IO displacement, protruding difference of zygoma, and facial asymmetry index of group 1 were $2.15 \pm 1.48,4.07 \pm 2.22,3.50 \pm 2.94$, and $5.82 \pm 2.42$, respectively, and those of group 2 were $2.47 \pm 2.26, \quad 5.24 \pm 3.55,2.50 \pm 1.39$, and $4.84 \pm 2.21$. No variable was statistically different between groups (Table 2). On the other hand, all variables were significantly different before and after surgery within each group (Table 3 ). Moreover, the means of operation time, hospital stay, and follow-up period of group 1 were $96.25 \pm 26.07,6.00 \pm 1.59$, and $63.35 \pm 35.54$, respectively, and those of group 2 were $116.02 \pm 28.50,5.45 \pm 1.00$, and $93.50 \pm 121.20$. Operation 


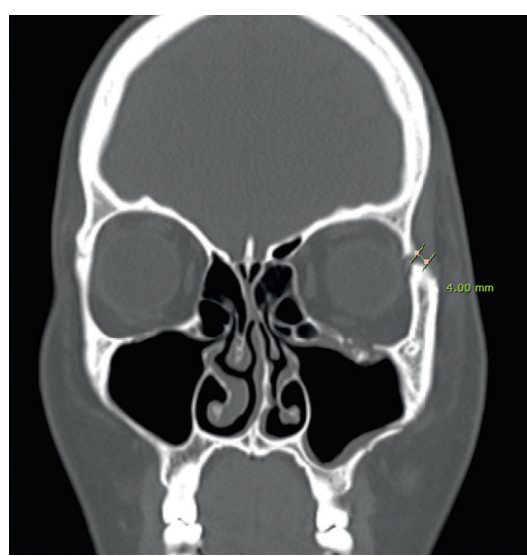

(a)

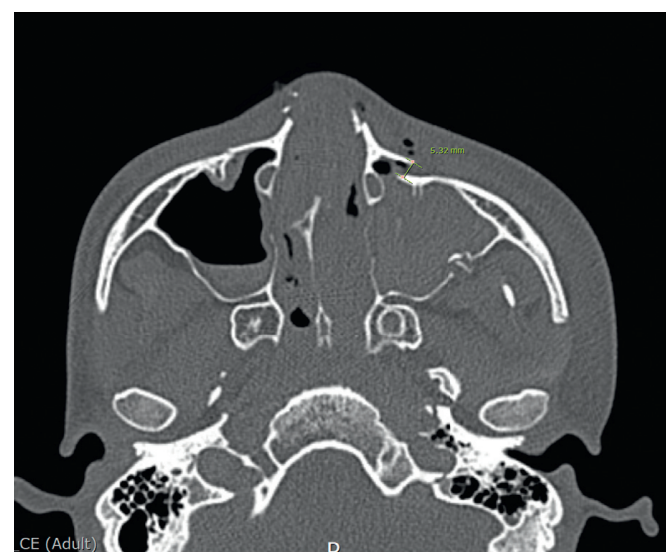

(b)

FIGURE 1: Degree of reduction. Gauging displacement distance between outer cortical bones. (a) Measurement of the cortical gap of the zygomaticofrontal suture. (b) Measurement of the cortical gap of the inferior orbital rim.

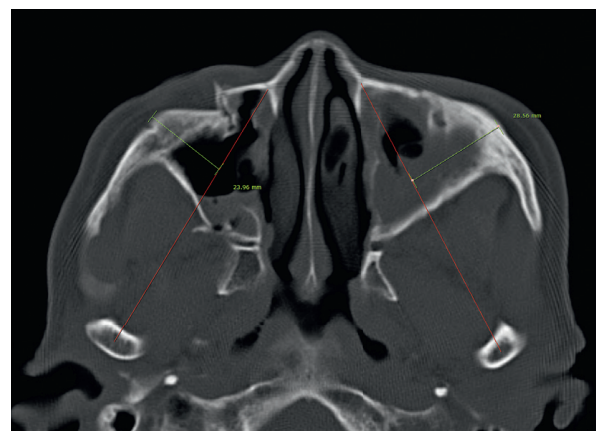

(a)

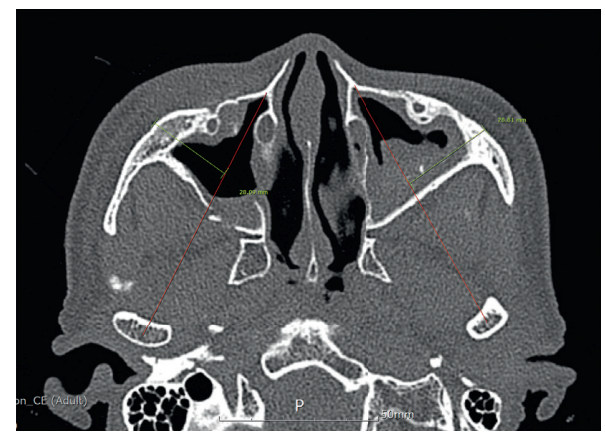

(b)

FIGURE 2: The protruding difference of zygoma: compared by measuring the distance from the most prominent point of each zygomatic arch to A line (A line: a virtual line from the pyriform aperture to the condyle of the mandible). (a) Preoperative measurement. (b) Postoperative measurement.

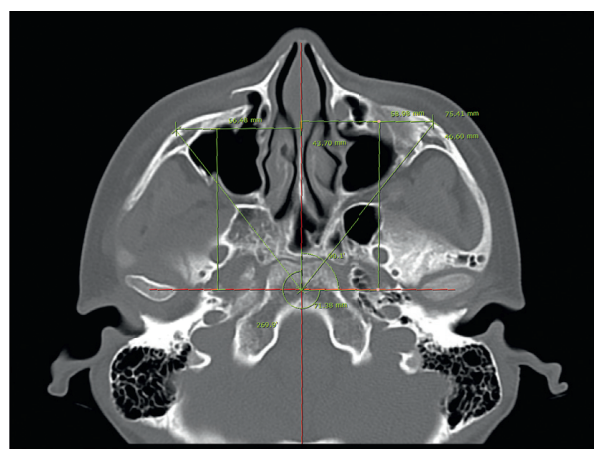

(a)

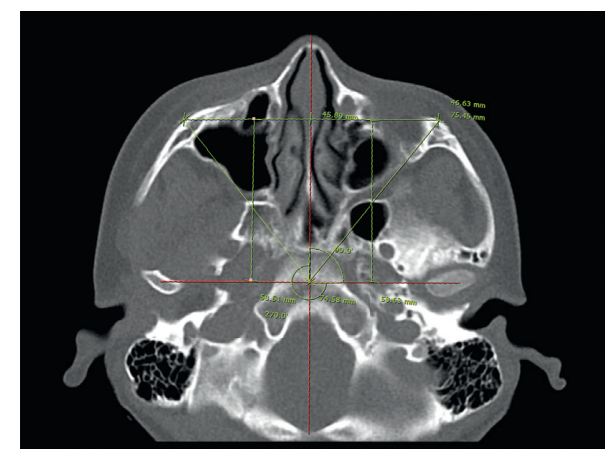

(b)

FIgURE 3: The asymmetry index of zygomatic prominence: comparing the difference in the two prominences of the zygomatic bone. (a) Preoperative measurement. (b) Postoperative measurement (Hr: right horizontal length, Hl: left horizontal length, Vr: right vertical length, Vl: left vertical length, Dr: right distance from midpoint, and Dl: left distance from midpoint). Asymmetry index $=\sqrt{(\mathrm{Hr}-\mathrm{Hl})^{2}+(\mathrm{Vr}-\mathrm{Vl})^{2}+(\mathrm{Dr}-\mathrm{Dl})^{2}}$.

time was significantly different between groups $(P$ value: 0.026), but average duration of hospital stay and follow-up period were similar ( $P$ values: 0.338 and 0.763 , respectively) (Table 4).

\section{Discussion}

The results showed that preoperative and postoperative variables of the two groups were not statistically different, 
Table 1: Patient characteristics and demographic data.

\begin{tabular}{|c|c|c|c|}
\hline & Group 1 & Group 2 & $P$ value \\
\hline Age, year & $45.40 \pm 21.36$ & $53.10 \pm 20.57$ & 0.253 \\
\hline Sex & & & 0.354 \\
\hline Male & $13(65 \%)$ & $14(70 \%)$ & \\
\hline Female & $7(35 \%)$ & $6(30 \%)$ & \\
\hline Lesion side & & & 0.642 \\
\hline Right & $6(30 \%)$ & $8(40 \%)$ & \\
\hline Left & $14(70 \%)$ & $12(60 \%)$ & \\
\hline Cause & & & 0.510 \\
\hline Traffic accident & $2(10 \%)$ & $4(20 \%)$ & \\
\hline Fall down & $9(45 \%)$ & $11(55 \%)$ & \\
\hline Assault & $3(15 \%)$ & $2(10 \%)$ & \\
\hline Accidental bump & $5(25 \%)$ & $3(15 \%)$ & \\
\hline Concomitant injuries & $3(15 \%)$ & $4(20 \%)$ & 0.509 \\
\hline Operation delay, day & $9.60 \pm 3.65$ & $10.10 \pm 4.24$ & 0.692 \\
\hline
\end{tabular}

TABLE 2: Surgical outcomes: comparison of group 1 and group 2.

\begin{tabular}{llll}
\hline & Group 1 & Group 2 & $P$ value \\
\hline Preoperative variables $(\mathrm{mm})$ & & & \\
ZF displacement & $2.15 \pm 1.48$ & $2.47 \pm 2.26$ & \\
IO displacement & $4.07 \pm 2.22$ & $5.24 \pm 3.55$ & 0.603 \\
Protruding difference of zygoma & $3.50 \pm 2.94$ & $2.50 \pm 1.39$ & 0.383 \\
Asymmetry index & $5.82 \pm 2.42$ & $4.84 \pm 2.21$ & \\
Preoperative variables (mm) & & & \\
ZF displacement & $1.25 \pm 1.13$ & $1.48 \pm 1.24$ & 0.189 \\
IO displacement & $1.55 \pm 1.55$ & $0.85 \pm 1.24$ & 0.556 \\
Protruding difference of zygoma & $1.53 \pm 1.60$ & $1.64 \pm 1.11$ & 0.136 \\
Asymmetry index & $2.35 \pm 0.85$ & $2.43 \pm 0.85$ & 0.324 \\
\hline
\end{tabular}

TABLE 3: Surgical outcomes: comparison of preoperative and postoperative variables within each group.

\begin{tabular}{lccc}
\hline & Preoperative & Postoperative & $P$ value \\
\hline Variable of group 1 $(\mathrm{mm})$ & & & \\
ZF displacement & $2.15 \pm 1.48$ & $1.25 \pm 1.13$ & $0.006^{*}$ \\
IO displacement & $4.07 \pm 2.22$ & $1.55 \pm 1.55$ & $<.001^{* * *}$ \\
Protruding difference of zygoma & $3.50 \pm 2.94$ & $1.53 \pm 1.60$ & $0.012^{*}$ \\
Asymmetry index & $5.82 \pm 2.42$ & $2.35 \pm 0.85$ & $<0.0001^{* * * *}$ \\
Variable of group 2 (mm) & & & $0.022^{*}$ \\
ZF displacement & $2.47 \pm 2.26$ & $1.48 \pm 1.24$ & $<0.0001^{* * * *}$ \\
IO displacement & $5.24 \pm 3.55$ & $0.85 \pm 1.24$ & $0.024^{*}$ \\
Protruding difference of zygoma & $2.50 \pm 1.39$ & $1.64 \pm 1.11$ & $<0.0001^{* * * *}$ \\
Asymmetry index & $4.84 \pm 2.21$ & $2.43 \pm 0.85$ & \\
\hline
\end{tabular}

TABle 4: Operation time, hospitalization, and follow-up period.

\begin{tabular}{lccc}
\hline & Group 1 & Group 2 & $P$ value \\
\hline Operation time, minute & $96.25 \pm 26.07$ & $116.02 \pm 28.50$ & $0.026^{*}$ \\
Hospital stay, day & $6.00 \pm 1.59$ & $5.45 \pm 1.00$ & 0.338 \\
Follow-up period, day & $63.35 \pm 35.54$ & $93.50 \pm 121.20$ & 0.763 \\
\hline
\end{tabular}

while the variables before and after surgery within each group were significantly changed. In other words, the surgical results of the two groups were the same, despite one fewer incision used to access the $\mathrm{ZF}$ of group 1 . The altered two-point fixation, excluding an incision approaching the $\mathrm{ZF}$, required a shorter operation time, allowing a more efficient surgery than three-point fixation of ZMC fracture.
The primary goal of this study is to avoid lateral brow incision and ORIF of the ZF suture to achieve reconstruction of ZMC fracture without sequelae of unnecessary scar, palpation of plates, and ectropion $[8,12,16]$. In addition, the operation time is shortened. Instead, the standard of anatomical alignment is based on the surface of the orbital floor and the continuity of IO rim in the transverse direction and 
the ZM complex as the basis of the vertical buttress. Although transconjunctival incision at the upper eyelid has been tried to avoid transcutaneous incisions for ZF sutures [20], it has not been generally accepted. One study attempted to select ORIF sites other than the ZF suture [16], but it focused on patient satisfaction without quantitative analysis. In the present study, statistical analysis and a follow-up investigation of the results are clearly presented.

In the traditional three-point ORIF, ZF, IO, and ZM have been essential points for achieving stability in a ZMC fracture $[2,15,21]$ and have been approached through lateral brow, subciliary or transconjunctival, and intraoral incisions, respectively. However, approaches via three points require a long surgery time and may result in complications including ectropion and noticeable scars [22]. Although selection of ORIF with fewer than three points has been studied, most techniques include the ZF suture [2, 5, 6, 9-15, 22].

The zygomatic bone occupies the most prominent of malar eminences, and it forms the facial width and a major buttress of the midface [2]. It has a tetrapod structure composed of 4 articulations, referred to as the $\mathrm{ZM}, \mathrm{ZF}$, zygomaticotemporal (ZT), and ZS sutures [7, 23]. ZMC fracture, including all tetrapod, is the second most common facial bone fracture $[7,24]$ and may result in critical deformity $[3,4]$. Accuracy of ZMC reconstruction is essential to restore orbital volume and to reestablish facial projection and width [25].

The classification of the present study distinguishes three types of ZMC fractures: A, B, and C [17]. According to this classification, type A injury means the fracture of isolated one component of the buttress, such as the zygomatic arch (type A1), the lateral orbital wall (type A2), and the inferior orbital rim (type A3). Type B fracture includes all four buttresses, so-called tetrapod fracture which was the indication of the surgery in the present study. Type C injury is classified as complex fractures with comminution of the zygomatic bone. The indication of the ORIF in the present study was only type B ZMC fracture.

Fracture healing is the process in which bony tissue restores its innate physical and mechanical properties [26]. In the beginning 4 to 6 weeks of bone healing, the callus is frail, and mechanical stability is a crucial factor to form an appropriate callus by means of external or internal fixation. This results in gradual maturation of the callus from woven to lamellar bone [27]. If fixation is not successful, the callus may not be calcified, and an unstable fibrous union may be generated [26]. Therefore, rigid or semirigid fixation of fractured bone is essential, and the fixation by absorbable plates and screw provides good long-term stability to achieve the healing of the ZMC fracture [28-33].

The standard treatment of ZMC fracture is ORIF [5]; except for the ZT suture, the locations and number of fixation points remain in dispute $[6,16]$. Because isolated fractures of the ZT complex or zygomatic arch are often mild $[1,6]$, closed reduction is the effective treatment through the Gillies approach or Keen's approach [6,34]. Gillies incision is no more than $2.5 \mathrm{~cm}$ parallel to the hair follicles through the temporal scalp within the hairline, and the Dingman elevator passes between the deep fascia and the temporalis muscle $[1,23]$.
The ZF complex is a narrow and dense bony region that acts as the lateral vertical maxillary buttress and is a reliable site to secure stability [9] and to evaluate well-reduced alignment without rotational deformity of the ZS suture [8]. The ZF complex is thus most commonly selected for fixation $[2,5,6,10-15]$. A Dingman elevator can be inserted under the zygomatic arch for effective reduction through upper eyelid, lateral brow, and extended lower eyelid incisions [35-38]. However, ORIF of the ZF suture accompanying these incisions often produces sequelae, including a striking scar, unpleasant perception of plates via thin skin, ectropion, and risk of injury to the anterior cranial fossa $[8,12,16]$.

The IO has a role in the upper transverse maxilla with the ZM complex across the ZT suture $[8,23]$, and it can be accessed through numerous incisions including subciliary, subtarsal, intraorbital, and transconjunctival $[5,23,39]$. In cases of diplopia, enophthalmos, and comminuted fracture, the inferior orbital rim can be explored simultaneously to evaluate accompanying impure blow-out fracture [40]. Furthermore, this study suggests that exploring the inferior orbital wall and alignment of the IO can be indicators of successful anatomical alignment. In group 2, in the immediate postoperative reduction state and postoperative radiological test, evaluating the concave surface of the orbital floor and the straightened continuity of the IO was a reliable, acceptable standard.

The ZM and pterygomaxillary complexes are the major vertical buttresses for mastication [10] and unite the maxillary alveolus with the ZT complex. Keen's approach is an intraoral route using a mucosal incision [23] to expose ZM and pterygomaxillary buttresses $[1,41,42]$. This approach can be used in closed reduction through the stab incision.

Lateral brow incision permits a Dingman approach, which provides the strongest rotary force on the ZMC fracture segment from the caudal direction in the vertical axis. The present study used only Keen's approach, involving a dual-top screw for restoration in severe displacement or impaction. Dual-top screws are useful especially in cases where the fractured fragment is displaced downward and rotated inward without excessive reduction force [41, 43]. As it is unnecessary to expose the buttress widely or to separate zygomatic segments from soft tissue and muscle in this procedure, the rate of soft tissue complications including cheek drooping is low.

Other methods to overcome the inadequate vector for reduction of the zygoma are Kirschner's wire $[44,45]$ and T-bar screw $[15,46]$ traction. Both methods allow precise three-dimensional manipulation of a fractured segment, after which reduction is controllable in any vector and direction can involve an elevator via Keen's approach.

The limitations of the present study are that it is not a randomized controlled trial, but the retrospective study. And the exact measurement point of zygomatic redirection cannot be defined. Also, the number of participating patients was small.

\section{Conclusion}

Altered two-point fixation of zygomaticomaxillary complex fracture, excluding an incision approaching the $\mathrm{ZF}$, provides 
surgical efficacy. The method presents the same surgical outcomes as traditional three-point fixation. However, because altered two-point fixation includes one less incision, it requires less operation time and reduces the noticeable scar and complications of a palpable and exposed plate via the skin.

\section{Data Availability}

The data from CT measurement used to support the findings of this study are available from the corresponding author upon request.

\section{Conflicts of Interest}

The authors declare that there are no conflicts of interest regarding the publication of this paper.

\section{Acknowledgments}

This research was supported by a grant of the Korea Health Technology R\&D Project through the Korea Health Industry Development Institute (KHIDI), funded by the Ministry of Health \& Welfare, Republic of Korea (HI18C2174). And, The author wish to acknowledge the financial support of the Catholic Medical Center Research Foundation made in the program year of 2016 (5-2016-B0001-00163).

\section{References}

[1] B. G. A. Evans and G. R. D. Evans, "MOC-PSSM CME article: zygomatic fractures," Plastic and Reconstructive Surgery, vol. 121, pp. 1-11, 2008.

[2] A. Chakranarayan, G. K. Thapliyal, R. Sinha, and M. P. Suresh, "Efficacy of two point rigid internal fixation in the management of zygomatic complex fracture," Journal of Maxillofacial and Oral Surgery, vol. 8, no. 3, pp. 265-269, 2009.

[3] R. Rohrich, L. H. Hollier, and D. Watumull, "The orbital apex syndrome: compromised vision associated with high-velocity orbitozygomatic fractures," Seminars in Plastic Surgery, vol. 6, no. 1, pp. 149-154, 1992.

[4] E. Ellis III and W. Kittidumkerng, "Analysis of treatment for isolated zygomaticomaxillary complex fractures," Journal of Oral and Maxillofacial Surgery, vol. 54, no. 4, pp. 386-400, 1996.

[5] Y. Yonehara, S. Hirabayashi, M. Tachi, and H. Ishii, "Treatment of zygomatic fractures without inferior orbital rim fixation," Journal of Craniofacial Surgery, vol. 16, no. 3, pp. 481-485, 2005.

[6] M. Eski, I. Sahin, M. Deveci, M. Turegun, S. Isik, and M. Sengezer, "A retrospective analysis of 101 zygomaticoorbital fractures," Journal of Craniofacial Surgery, vol. 17, no. 6, pp. 1059-1064, 2006.

[7] D. Meslemani and R. M. Kellman, "Zygomaticomaxillary complex fractures," Archives of Facial Plastic Surgery, vol. 14, no. 1, pp. 62-66, 2012.

[8] P. Kelley, R. Hopper, and J. Gruss, "Evaluation and treatment of zygomatic fractures," Plastic and Reconstructive Surgery, vol. 120, no. 2, pp. 5S-15S, 2007.

[9] D. Rohner, A. Tay, C. S. Meng, D. W. Hutmacher, and B. Hammer, "The sphenozygomatic suture as a key site for osteosynthesis of the orbitozygomatic complex in panfacial fractures: a biomechanical study in human cadavers based on clinical practice," Plastic and Reconstructive Surgery, vol. 110, no. 6, pp. 1463-1471, 2002.

[10] M. Rana, R. Warraich, S. Tahir et al., "Surgical treatment of zygomatic bone fracture using two points fixation versus three point fixation-a randomised prospective clinical trial," Trials, vol. 13, no. 1, p. 36, 2012.

[11] S. Olate, S. M. Lima Jr., R. Sawazaki, R. W. F. Moreira, and M. de Moraes, "Surgical approaches and fixation patterns in zygomatic complex fractures," Journal of Craniofacial Surgery, vol. 21, no. 4, pp. 1213-1217, 2010.

[12] B. R. Chrcanovic, Y. S. L. Cavalcanti, and P. Reher, "Temporal miniplates in the frontozygomatic area-an anatomical study," Oral and Maxillofacial Surgery, vol. 13, no. 4, pp. 201-206, 2009.

[13] P.-K. Lee, J.-H. Lee, Y.-S. Choi et al., "Single transconjunctival incision and two-point fixation for the treatment of noncomminuted zygomatic complex fracture," Journal of Korean Medical Science, vol. 21, no. 6, pp. 1080-1085, 2006.

[14] C. Mohammadinezhad, "Evaluation of a single miniplate use in treatment of zygomatic bone fracture," Journal of Craniofacial Surgery, vol. 20, no. 5, pp. 1398-1402, 2009.

[15] S. H. Song, H. Kwon, S.-H. Oh, S.-J. Kim, J. Park, and S. I. Kim, "Open reduction of zygoma fractures with the extended transconjunctival approach and T-bar screw reduction," Archives of Plastic Surgery, vol. 45, no. 4, pp. 325332, 2018.

[16] S. T. Kim, D. H. Go, J. H. Jung, H. E. Cha, J. H. Woo, and I. G. Kang, "Comparison of 1-point fixation with 2-point fixation in treating tripod fractures of the zygoma," Journal of Oral and Maxillofacial Surgery, vol. 69, no. 11, pp. 2848-2852, 2011.

[17] M. Zingg, K. Laedrach, J. Chen et al., "Classification and treatment of zygomatic fractures: a review of 1,025 cases," Journal of Oral and Maxillofacial Surgery, vol. 50, no. 8, pp. 778-790, 1992.

[18] M. S. Khaqani, F. Tavosi, M. Gholami, H. R. Eftekharian, and L. Khojastepour, "Analysis of facial symmetry after zygomatic bone fracture management," Journal of Oral and Maxillofacial Surgery, vol. 76, no. 3, pp. 595-604, 2018.

[19] F. Ras, L. L. Habets, F. C. van Ginkel, and B. Prahl-Andersen, "Method for quantifying facial asymmetry in three dimensions using stereophotogrammetry," The Angle Orthodontist, vol. 65, no. 3, pp. 233-239, 1995.

[20] P. R. Langsdon, G. T. Rohman, R. Hixson, M. R. Stumpe, and S. E. Metzinger, "Upper lid transconjunctival versus transcutaneous approach for fracture repair of the lateral orbital rim," Annals of Plastic Surgery, vol. 65, no. 1, pp. 52-55, 2010.

[21] G. C. Rinehart, J. L. Marsh, K. M. Hemmer, and S. Bresina, "Internal fixation of malar fractures," Plastic and Reconstructive Surgery, vol. 84, no. 1, pp. 21-25, 1989.

[22] T. Jo and J. Kim, "An anthropometric and three-dimensional computed tomographic evaluation of two-point fixation of zygomatic complex fractures," Archives of Plastic Surgery, vol. 41, no. 5, pp. 493-499, 2014.

[23] M. R. Markiewicz, S. Gelesko, and R. B. Bell, "Zygoma reconstruction," Oral and Maxillofacial Surgery Clinics of North America, vol. 25, no. 2, pp. 167-201, 2013.

[24] D. S. Covington, D. J. Wainwright, J. F. Teichgraeber, and D. H. Parks, "Changing patterns in the epidemiology and treatment of zygoma fractures," The Journal of Trauma: Injury, Infection, and Critical Care, vol. 37, no. 2, pp. 243-248, 1994. 
[25] K. Bogusiak and P. Arkuszewski, "Characteristics and epidemiology of zygomaticomaxillary complex fractures," Journal of Craniofacial Surgery, vol. 21, no. 4, pp. 1018-1023, 2010.

[26] I. H. Kalfas, "Principles of bone healing," Neurosurgical Focus, vol. 10, no. 4, pp. 1-4, 2001.

[27] P. V. Giannoudis, T. A. Einhorn, and D. Marsh, "Fracture healing: the diamond concept," Injury, vol. 38, no. 4, 2007.

[28] R. R. M. Bos, G. Boering, F. R. Rozema, and J. W. Leenslag, "Resorbable poly(L-lactide) plates and screws for the fixation of zygomatic fractures," Journal of Oral and Maxillofacial Surgery, vol. 45, no. 9, pp. 751-753, 1987.

[29] R. B. Bell and C. S. Kindsfater, "The use of biodegradable plates and screws to stabilize facial fractures," Journal of Oral and Maxillofacial Surgery, vol. 64, no. 1, pp. 31-39, 2006.

[30] R. Suuronen, "Biodegradable fracture-fixation devices in maxillofacial surgery," International Journal of Oral and Maxillofacial Surgery, vol. 22, no. 1, pp. 50-57, 1993.

[31] D. E. Cutright, E. E. Hunsuck, and J. D. Beasley, "Fracture reduction using a biodegradable material, polylactic acid," Journal of Oral Surgery, vol. 29, no. 6, pp. 393-397, 1971.

[32] R. Suuronen, P. Haers, C. Lindqvist, and H. Sailer, "Update on bioresorbable plates in maxillofacial surgery," Facial Plastic Surgery, vol. 15, no. 1, pp. 61-72, 1999.

[33] S. Degala, S. Shetty, and S. Ramya, "Fixation of zygomatic and mandibular fractures with biodegradable plates," Annals of Maxillofacial Surgery, vol. 3, no. 1, pp. 25-30, 2013.

[34] W. M. M. T. van Hout, E. M. Van Cann, R. Koole, and A. J. W. P. Rosenberg, "Surgical treatment of unilateral zygomaticomaxillary complex fractures: a 7-year observational study assessing treatment outcome in 153 cases," Journal of Cranio-Maxillofacial Surgery, vol. 44, no. 11, pp. 1859-1865, 2016.

[35] D. S. Kung and L. B. Kaban, "Supratarsal fold incision for approach to the superior lateral orbit," Oral Surgery, Oral Medicine, Oral Pathology, Oral Radiology, and Endodontology, vol. 81, no. 5, pp. 522-525, 1996.

[36] B. L. Eppley, P. L. Custer, and A. M. Sadove, "Cutaneous approaches to the orbital skeleton and periorbital structures," Journal of Oral and Maxillofacial Surgery, vol. 48, no. 8, pp. 842-854, 1990.

[37] Z. W. Pozatek, L. B. Kaban, and W. C. Guralnick, "Fractures of the zygomatic complex: an evaluation of surgical management with special emphasis on the eyebrow approach," Journal of Oral Surgery, vol. 31, no. 2, pp. 141-148, 1973.

[38] W. D. Appling, J. R. Patrinely, and T. A. Salzer, "Transconjunctival approach vs subciliary skin-muscle flap approach for orbital fracture repair," Archives of Otolaryngology-Head and Neck Surgery, vol. 119, no. 9, pp. 1000-1007, 1993.

[39] A. Baumann and R. Ewers, "Transcaruncular approach for reconstruction of medial orbital wall fracture," International Journal of Oral and Maxillofacial Surgery, vol. 29, no. 4, pp. 264-267, 2000.

[40] K. Shumrick and A. Campbell, "Management of the orbital rim and floor in zygoma and midface fractures: criteria for selective exploration," Facial Plastic Surgery, vol. 14, no. 1, pp. 77-81, 1998.

[41] S.-H. Moon, J.-H. Lee, D.-Y. Oh, J.-W. Rhie, and S.-T. Ahn, "Reduction of zygomatic fracture segment with intermaxillary fixation screw," Journal of Craniofacial Surgery, vol. 23, no. 3, pp. 842-844, 2012.

[42] M. A. Shikara, K. T. Vakharia, and J. D. Greywoode, "Zygomaticomaxillary complex-orbit fracture alignment," JAMA Facial Plastic Surgery, vol. 20, no. 5, pp. 428-429, 2018.
[43] B. O’Regan and M. Devine, "Screw-wire traction technique: aid to anatomical reduction of multi-segment mid-facial fractures," British Journal of Oral and Maxillofacial Surgery, vol. 51, no. 5, pp. 459-460, 2013.

[44] M. Bezuhly, J. Lalonde, M. Alqahtani, G. Sparkes, and D. H. Lalonde, "Gillies elevation and percutaneous Kirschner wire fixation in the treatment of simple zygoma fractures: long-term quantitative outcomes," Plastic and Reconstructive Surgery, vol. 121, no. 3, pp. 948-955, 2008.

[45] B.-Y. Y. Park, S. Y. Song, I. S. Yun, D. W. Lee, D. K. Rah, and W. J. Lee, "First percutaneous reduction and next external suspension with Steinmann pin and Kirschner wire of isolated zygomatic fractures," Journal of Craniofacial Surgery, vol. 21, no. 4, pp. 1060-1065, 2010.

[46] E. L. Chang, M. P. Hatton, C. R. Bernardino, and P. A. D. Rubin, "Simplified repair of zygomatic fractures through a transconjunctival approach," Ophthalmology, vol. 112, no. 7, pp. 1302-1309, 2005. 